Original Research Article

\title{
Prospective observational study of drug utilization in neonatal seizure at a tertiary care hospital with Pharmacoeconomics
}

\author{
Ketan A. Patil ${ }^{1}$, Santoshkumar R. Jeevangi ${ }^{2}$, Sharanabasappa ${ }^{3 *}$
}

${ }^{1}$ Department of Pharmacology, All India Institute of Medical Sciences, Jodhpur, Rajasthan, India

${ }^{2}$ Department of Pharmacology, Mahadevappa Rampure Medical College, Kalaburagi, Karnataka, India

${ }^{3}$ Department of Pharmacology, AECS Maaruti Dental College, Bangalore, Karnataka, India

Received: 08 February 2019

Accepted: 12 February 2019

\section{*Correspondence to:}

Dr. Sharanabasappa,

Email: sharan2014@gmail.com

Copyright: (C) the author(s), publisher and licensee Medip Academy. This is an openaccess article distributed under the terms of the Creative Commons Attribution NonCommercial License, which permits unrestricted noncommercial use, distribution, and reproduction in any medium, provided the original work is properly cited.

\begin{abstract}
Background: Seizures are the most common indicator of significant neurologic dysfunction in neonatal period with incidence of 11.7/1000 live births. Phenobarbitone is used as first line of treatment since 1900s. Newer anti-epileptic drugs (AED) available are Levetiracetam, Topiramate etc. Present study focused on utilization pattern of AED, treatment outcomes and to study economic burden of disease.

Methods: An observational study was done on 100 neonates admitted to Neonatal Intensive Care Unit in Basaveshwara hospital, Kalaburagi (June 2016May 2017). Prescription data was entered into specially designed proforma, WHO core indicators were determined. The data was analyzed using descriptive statistics and presented as means and percentages.

Results: Majority of neonates were male $(58 \%)$ and $63 \%$ were diagnosed with subtle seizure. Out of 458 drugs prescribed, 201 were antiepileptics. $41 \%$ cases were successfully managed by monotherapy. Most commonly used drug was phenobarbitone $(82 \%)$ and phenytoin $(31 \%)$. Leviteracetam, newer AED was used in 3 refractory cases. The major combination of drugs used was Phenobarbitone-Phenytoin (24\%). AED were rationally prescribed, but antibiotic was over-utilized(68\%). $31 \%$ cases had adverse drug reaction. On average per prescription, number of drugs used were 4.6 and drug cost was Rs.3803/-. The total cost of illness per patient was Rs.16363/-.

Conclusions: AED utilization in neonatal seizures was in accordance to guidelines, with phenobarbitone being extensively used despite its potential neurotoxicity. The utilization of newer AED would increase if clinicians are supported with the safety and efficacy data. Although monotherapy was preferred with respect to AED, antibiotics were highly prescribed; hence awareness is needed to curb this practice.
\end{abstract}

Keywords: Cost of illness, Drug utilization, Monotherapy, Neonatal seizures, Phenobarbitone

\section{INTRODUCTION}

Drugs or substances that modify the function of body have been challenging the intellect of human beings since ancient times. In a race to discover better ways of survival, man has left no stones unturned to find drugs to keep him alive. There are more effective drugs today on the market than ever before. Yet, drugs are frequently not used to their full potential or according to the generally accepted criteria. Drug utilization studies lights up the path in this aspect.

Seizure refers to a transient alteration of behavior due to the disordered, synchronous and rhythmic firing of populations of brain neurons. ${ }^{1}$ A seizure in the neonatal period is a medical emergency indicating significant insult to the immature brain, and thus requires prompt diagnostic, therapeutic interventions and treatment. 
Neonatal mortality rate in India is as high as $29 / 1000$ live births. Mortality is highest during the 1 st $24 \mathrm{hrs}$ after birth. Neonatal seizure accounts for $18 \%$ of overall neonatal morbidity. The immature brain seems more prone to seizures than a mature brain. ${ }^{2}$ Antiepileptic drug options available are phenobarbitone, phenytoin, sodium valproate and lorazepam. Newer drugs like topiramate shows potential neuro-protective effect against injury caused by seizures and levetiracetam has little drug-to-drug interactions. $^{3}$

Drug utilization studies are useful to provide denominators to calculate rates of reported adverse drug reactions, to monitor the utilization of drugs from therapeutic categories where particular problems can be anticipated. ${ }^{4}$ Prescribing practices are a reflection of health professional's abilities to choose among the various drugs and determine the ones that will benefit the patients. Pediatric age group, particularly neonatal period, is a vulnerable group in whom rational prescription of drugs plays a vital role especially in disease like seizures affecting CNS which determines the future of the child. Treatment options for neonatal seizures remain limited despite the fact that several newer antiepileptic medications have been introduced in market over the last two decades.

On one hand this newer antiepileptic has to prove their efficacy in neonates, the older ones have proven their efficacy with some serious side effects. In such scenarios the clinical decision becomes difficult. Without knowledge of how drugs are being prescribed and used, it is difficult to comment on rational drug use or to suggest improvements in prescribing habits. Thus, drug utilization studies are the linchpin of any auditing system. Hence the present study was undertaken to assess management practices for seizures in neonates along with cost of illness analysis in neonatal seizures in NICU of a tertiary care hospital.

\section{METHODS}

This prospective and observational study was done after taking permission from Institute Ethics Committee, Mahadevappa Rampure Medical College, Kalaburagi, Karnataka, India.

\section{Inclusion criteria}

Neonates diagnosed with neonatal seizure in NICU of Basaveshwara Teaching and General Hospital were included in the study.

\section{Exclusion criteria}

- Neonates weighting less than $800 \mathrm{gms}$,

- Incredibly LBW babies,

- Child born to HIV or HBsAg +ve mother,

- Child born with cardiac defects,

- Chromosomal abnormalities
After obtaining informed consent from the parent or guardian, data were collected for demographics, diagnosis, investigations, treatment, outcome, ADR and duration of stay in a specially designed case record form.

Data was analyzed under the following parameters: total number of drugs prescribed, generic / brand name, most commonly prescribed drug, whether the drug is included in the essential drug list, cost of illness - to study the economic burden of the disease and adverse drug reaction monitoring. The WHO Core Indicator - drug use indicators were also determined.

\section{Statistical analysis}

The data collected were analyzed statistically using descriptive statistics. The result is expressed in form of mean and standard deviation. Wherever necessary the results will be depicted in the form of percentage and graphs. Microsoft Excel and Statistical Package for Social Sciences (SPSS) were used for statistical analysis.

\section{RESULTS}

Out of 100 neonates enrolled in the study, 58 were male and 42 were females. The study shows that $48 \%$ of neonates with seizure occurred on day 1 , whereas $77 \%$ neonates developed seizures within day 7 . The mean age of neonates in our study was 5.43 days. There were 27 neonates with co-morbid conditions along with neonatal seizures. Conditions like Muconium Aspiration Syndrome (9) and respiratory distress (6) topped the list of co-morbid condition (Figure 1).

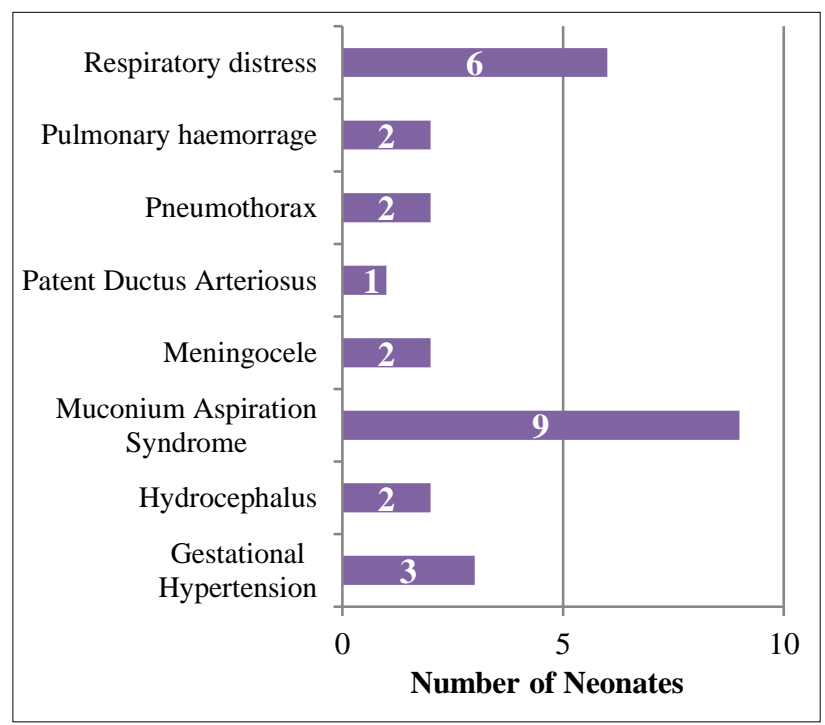

Figure 1: Co-morbid conditions.

In the present study there is predominance of term neonates which were $83 \%$ and $17 \%$ neonates were preterm. The mean gestational age in present study was $36.78 \pm 3.14$ weeks. There were $77 \%$ neonates with low birth weight. In present study $84 \%$ of cases had institutional deliveries. 
Majority of neonates i.e. $63 \%$ in the present study were diagnosed with subtle seizure. There were 22 cases of focal clonic seizure, 8 cases of multifocal and 7 cases of tonic seizures.

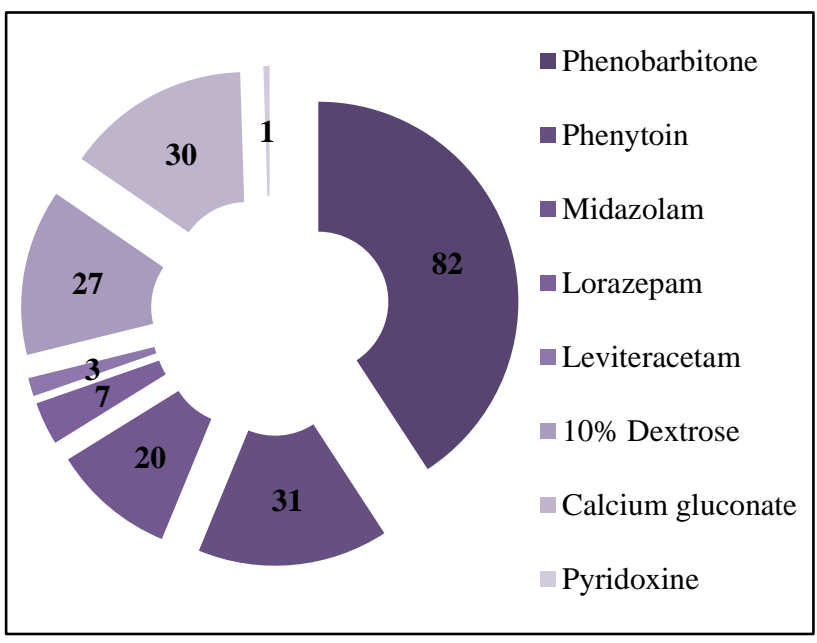

Figure 2: Different antiepileptics used.

In present study $41 \%$ of neonatal seizures were managed by single drug therapy. $73 \%$ of the drugs were being given through intravenous route. Out of total 458 drugs which were prescribed during the entire study, 201 were antiepileptic drugs. The most commonly used drug is phenobarbitone which is been given in $82 \%$ of neonate in the study followed by phenytoin in $31 \%$ of cases. In refractory cases benzodiazepines like midazolam (20\%) or lorazepam $(7 \%)$ were used. Leviteracetam, a newer antiepileptic drug was used in only 3 refractory cases (Figure 2). Other drugs used to control seizures were 10\% dextrose for hypoglycemic seizure and calcium gluconate for hypocalcaemia seizure. Supportive therapy with oxygen, antibiotics like ampicillin, gentamycin or meropenam and antipyretic paracetamol was used whenever necessary.

Table 1: Combination of antiepileptic drugs.

\begin{tabular}{|ll|}
\hline Combinations & Percentage (\%) \\
\hline Phenobarbitone + phenytoin & $24 \%$ \\
\hline Phenobarbitone + midazolam & $19 \%$ \\
\hline $\begin{array}{l}\text { Phenobarbitone + phenytoin }+ \\
\text { Midazolam }\end{array}$ & $6 \%$ \\
\hline $\begin{array}{l}\text { Phenobarbitone + phenytoin }+ \\
\text { levetiracetam }\end{array}$ & $1 \%$ \\
\hline $\begin{array}{l}\text { Phenobarbitone + midazolam }+ \\
\text { levetiracetam }\end{array}$ & $2 \%$ \\
\hline
\end{tabular}

The major combination of antiepileptic drug used was Phenobarbitone with phenytoin in $24 \%$ of cases (Table 1 ).

Total $89 \%$ of neonates were improved after the treatment. Two neonates succumbed to death after suffering from pulmonary hemorrhage. 30 neonates suffered from adverse drug reaction of which 17 were having sedation, 12 had respiratory depressions, 4 had hypotension and 7 neonates suffered from irritability. Polypharmacy was seen in all prescription. The average number of drugs per prescription is 4.6. Majority of cases were managed by $4-5$ drugs per prescription (Figure 3).

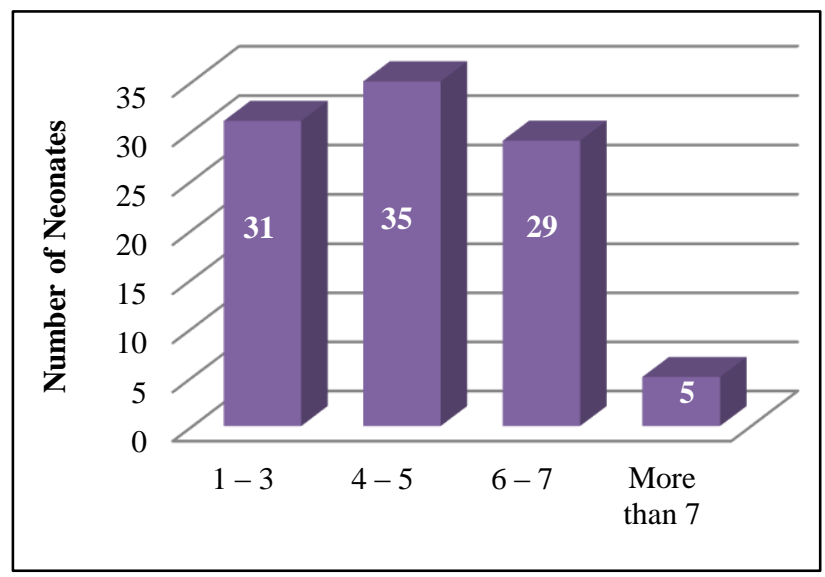

Figure 3: Number of drugs/ prescriptions.

Only $43 \%$ of drugs were prescribed by generic name which still needed to be improved as generic prescribing would rationalize the drug use and reduce the cost of drugs. Average consultation time was 16.3 minutes and average dispensing time is 4.8 minutes. Generally, longer consultation time had improved patient satisfaction and more effective use of resource.

The total cost of illness for neonatal seizures per patient in the present study is Rs.16363/-. Average drug cost per prescription is Rs.3803/-. The major cost that the guardians had to bear was cost of hospitalization amounting to Rs.9428/- (Figure 4). The average cost on injection was Rs.3613/- as majority of drugs were to be given through parenteral route due to serious condition of the neonate.

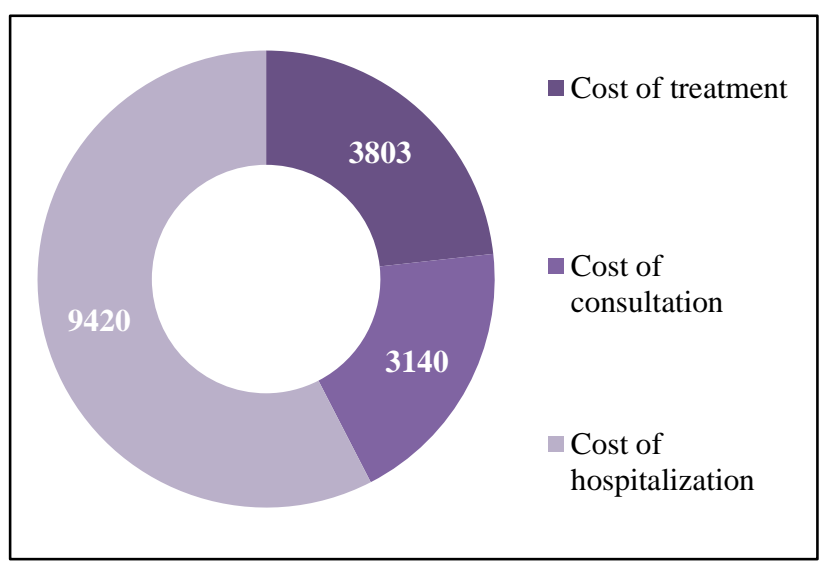

Figure 4: Cost of illness for neonatal seizures.

\section{DISCUSSION}

The enhanced excitability of neonatal brain makes them more vulnerable to seizure generation and poor response to 
conventional medications makes treating them a difficult task. Enhanced excitability of the neonatal brain is due to abundance of glutamanergic neurons with increased $\mathrm{N}$ methyl D-asparate (NMDA) and a-amino-3-hydroxy-5methyl-4-isoxazolepropionic acid (AMPA) receptor density. ${ }^{5}$

$y$ Amino Butyric Acid (GABA) in the immature brain has an excitatory function as the chloride gradient is reversed relative to the mature brain. This is predominantly due to expression of Sodium-Potassium-Chloride Cotransporter1 (NKCC1) in the neonatal period, leading to transport of $\mathrm{Cl}-$ into the cell at rest. ${ }^{6}$ With higher internal chloride concentrations channel opening leads to chloride leaving the cell and depolarizing it. With maturation, expression of NCCK1 decreases and Potassium Chloride Cotransporter2 (KCC2) increases which transports $\mathrm{Cl}-$ out of the cell and restores normalcy. ${ }^{7} \mathrm{KCC} 2$ is a critical mediator of synaptic inhibition and cellular protection against excitotoxicity.

Immature development of the excitatory and inhibitory neurotransmitter systems leads to a lack of good targets for conventional anti-seizure medications, which makes neonatal seizures particularly difficult to treat. The immature brain may be resistant to medications that act on GABA. This may be due to paradoxical chloride gradient as discussed above, lower receptor expression, and an immature receptor that is less sensitive to benzodiazepines than the adult brain. ${ }^{8}$

The incidence of seizures in infants born at term is $0.5-3$ per 1000 live births; the incidence is even higher in preterm infants, ranging from $1-13 \%$ of very low birth weight infants. ${ }^{9}$ Most of these epidemiological studies include only clinical seizures.

The exact incidence of electrographic, clinically silent seizures is yet unknown. The majority of neonatal seizures occur on the first day and $70 \%$ of all cases eventually recognized have been diagnosed by the fourth day. ${ }^{10}$ This finding was substantiated in present study as $48 \%$ neonatal seizures occurred on day 1 and $77 \%$ were diagnosed by day 7.

The overall incidence of neonatal seizures is 11.7 per 1000 live-births in India. The incidence increases with decreasing gestation age and birth weight. The incidence of seizures was $10.14 \%$ in very low birth weight babies in contrast to $0.59 \%$ with normal birth weight babies. ${ }^{11}$ In present study $77 \%$ neonates were having low birth weight which is similar finding in the study done by Lanska et al. ${ }^{12}$ The mean birth weight of neonates in our study was $2.16 \pm 0.51 \mathrm{kgs}$. The birth weight range was between 1.2 $3.5 \mathrm{kgs}$.

Similar to the study done by Omenga et al, there were $58 \%$ male and $42 \%$ female in this study. ${ }^{13}$ The male preponderance is found in neonatal seizure due to increased occurrence of reversal of chloride gradient due to $\mathrm{NKCC} 1$ channel.
The mean gestational age in present study was $36.78 \pm 3.14$ weeks. The gestational age range was between 26-41 weeks. This finding is in concordance with Park et al, which had $14 \%$ preterm and $86 \%$ term babies. ${ }^{14}$ Minchom $\mathrm{P}$ et al, found that post-term pregnancy, meconium stained amniotic fluid, prolonged second stage of labour, emergency caesarian section, assisted vaginal delivery, low Apgar score were significantly more common among cases of neonatal seizures. ${ }^{15}$ Similarly in present study meconium aspiration syndrome were frequently observed. Saliba RM et al, in their population-based study, evaluated risk factors for neonatal seizures and found that for preterm and term neonates, birth in a private or university hospital was a risk factor. ${ }^{16}$ Present study substantiates this fact as $84 \%$ of neonates were born in hospital premises.

Total $63 \%$ neonates in the present study were diagnosed with subtle seizure. This data correlates with study done by Calciolari $\mathrm{G}$ et al, having $65 \%$ of subtle seizures and $54 \%$ of multifocal clonic seizures as the most common seizure types. ${ }^{17} 22 \%$ of neonates have average NICU stay as $1-2$ days, $28 \%$ have 2 - 5 days, $36 \%$ have 5 - 10 days and $14 \%$ have more than 10 days in present stay. $50 \%$ of neonates with seizures were discharged within 5 days of admission.

A total of 458 drugs were prescribed in the entire study, out of which 201 were antiepileptic drugs. The major route of administration is intravenous with 334 drugs being given through intravenous route as it is an emergency condition. As condition of neonate improved the route of administration is switched over to oral on discharge.

Phenobarbitone although known for few of its side effects was prescribed in $82 \%$ of cases as it has proven its metal in treating neonatal seizures since a few decades, hence it has earning itself place to be on the top of the list of pediatrician or neonatologist. This was followed by phenytoin or fosphenytoin in $31 \%$ of cases especially when seizures were not controlled. In refractory cases drugs from other classes were prescribed like midazolam, lorazepam or leviteracetam.

Factors that treating pediatrician considered to select second line drugs were severity of neonatal condition, respiratory and cardiovascular status, presence or absence of cardiac, renal, or hepatic dysfunction and side effect profile of the drug.

Among the pool of newer antiepileptics, leviteracetam is quickly gaining the attention as it is readily available in both parenteral and enteral formulations, $66 \%$ of the drug is excreted unchanged in urine and the rest undergoes enzymatic hydrolysis to inactive metabolites without involving the hepatic cytochrome P450. ${ }^{18}$ Although it has a wide safety margin and efficacy reported in older infants and children the pharmacokinetic, safety profile and efficacy of levetiracetam for neonatal seizure are not fully understood and may differ from older children and adults. ${ }^{19}$ In present study levetiracetam was used in only 3 refractory cases owing to the above cited reasons. 
Among the 22 cases of metabolic derailment leading to seizures, $18 \%$ were having hypoglycaemia. The mean blood sugar level in hypoglycaemic cases is $32.70 \pm 4.7 \mathrm{mg} \%$. They were managed with $10 \%$ dextrose. The rest had hypocalcemia and managed by calcium gluconate. The major combination of drug used was Phenobarbitone with Phenytoin in $24 \%$ of cases followed by Phenobarbitone with Midazolam in $19 \%$ of cases.

Thirty neonates suffered from adverse drug reaction of which $17 \%$ were having sedation, $12 \%$ respiratory depression, $4 \%$ hypotension and $7 \%$ irritability. Sedation may be attributed to the use of phenobarbitone which inhibits GABA system. Respiratory depression may be attributed to either drugs or the condition of hypoxic brain injury the differentiation of which is clinically difficult.

The average number of drugs per prescription is 4.6. The average number of drugs per prescription in the present study was higher than the ideal WHO standard which is less than two (1.6-1.8). ${ }^{20}$ In present study $66 \%$ of neonates were prescribed less than 5 drugs. Brand prescribing is associated with increased treatment costs and bioequivalence problems.

Percentage of prescription with an antibiotic is $68 \%$ which is almost double the WHO standards (20-26.8\%). ${ }^{20}$ Warrier I et al, also found similar pattern of high rate of antibiotic exposure in their study. ${ }^{21}$ The most commonly prescribed antibiotics were ampicillin and gentamycin in $41 \%$ cases. This is probably due to the standard practice of administering antibiotics to pending bacterial culture results in sick neonates or providing prophylactic empirical antibiotic coverage to prevent any further complication in future. Although unavoidable in some clinical situation this indiscriminate and inadequate use of antibiotic can lead to resistance and a scenario of post antimicrobial era, where all the existing antimicrobial agents can be historical, will be expected in the near future. This is due to the imbalance between accelerating rate of antimicrobial resistance and decelerating rate of new antimicrobial drug development.

Percentage of encounters with an injection prescribed was $100 \%$ this is due to the need for early and rapid treatment. This finding is overestimation of actual prescribing pattern in the light of fact that prescribing injections for in-patient prescription is a common phenomenon. Percentage of drugs prescribed from essential drug list was $80 \%$ which was similar to finding in study done by Karande $\mathrm{S}$ et al, where $90 \%$ drugs were prescribed from essential drug list. $^{22}$ There were $80 \%$ of drugs that were prescribed from essential drug list available with the clinicians.

Only $28 \%$ of the parents or guardians had knowledge of correct dosage. As the study group is neonates who are dependent on caregivers or parents or guardians so the attending physicians or pharmacists should clearly explain the dosage schedule on discharge which would help in improving the effectiveness of the treatment. Average drug cost per prescription is Rs.3803/- The total cost of illness for neonatal seizures per patient in the present study is Rs.16363/-. The major share of patient's money went in for paying the cost of hospitalization of Rs.9420/-. This was due to the fact that neonates had to be admitted in NICU.

As all studies have some flaws, our study was no exception. The neonates were followed for short period of time; a longer duration of study would provide the long term safety data of the AED, as they are potentially neurotoxic. Direct medical cost like cost of the drug, hospitalization and consultation were calculated but direct non-medical costs like cost of investigations and indirect cost like cost of transportation were not calculated.

A seizure in the neonatal period requires prompt diagnostic and therapeutic interventions. Irrespective of immediate resuscitative attempts with antiepileptic drugs, the mere presence of neonatal seizures signifies substantial risk for neurodevelopmental of neonate. Utmost care was taken while rationally prescribing $\mathrm{AED}$, both in aspect of giving the right dose with respect to weight of neonate and preferring monotherapy over polytherapy.

Brand name prescription of drugs was present in this study. Therefore, more effort is to be invested to effectively avoid the problems of brand prescribing and to promote safe, cost effective and accessible generic drugs. Antibiotics were used in $68 \%$ of cases which is fairly high. This was partly because of the tender age of the participants involved and critical clinical condition through which they were suffering; which demanded the use of antibiotic to prevent further complication due to hospital infection. But eventually extensive use of antibiotics will create a dreadful scenario of antibiotic resistance and superbugs. Drug utilization studies should be conducted regularly to know the changes in prescription pattern and to promote rational prescribing.

The study suggests educational initiative towards newer antiepileptic drugs, formation of drug policy and hospital formulary based on National Essential drug list to reduce the drug cost and ensure rational use of medicines.

\section{ACKNOWLEDGEMENTS}

The Authors wish to extend their gratitude to professor Dr. B. V. Patil and Dr. S. H. Vardhamane for their help and moral support during the support, the colleague Dr. Amreen Saba for helping in the preparation of this manuscript and proof reading. Authors applaud the help in obtaining the cases and service done by Department of Pediatrics towards the neonates admitted in NICU.

Funding: No funding sources

Conflict of interest: None declared

Ethical approval: The study was approved by the Institutional Ethics Committee (IEC no. HKES/MRMCK/IEC/17/10/11) 


\section{REFERENCES}

1. McNamara JO. Pharmacotherapy of epilepsy. In: Brunton LL, Chabner BA, Knollman BC editors. Goodman \& Gilman's The Pharmacological Basis of Therapeutics, 12th edition. Newyork: McGrawHill;2011:583-608.

2. Ronen GM, Buckley D, Penney S, Streiner DL. Longterm prognosis in children with neonatal seizures: a population-based study. Neurol. 2007 Nov; 69(19):1816-1822.

3. Maggie Lo-Yee Yau, Eva Lai-Wah Fung, and Pak Cheung Ng. Response of levetiracetam in neonatal seizures. World J Clin Pediatr. 2015 Aug 8;4(3):45-9.

4. Helena Gama. Drug utilization studies. Med Files. 2008;22(2/3):69-74.

5. Rakhade SN, Jensen FE. Epileptogenesis in the immature brain: emerging mechanisms. Nat Rev Neurol. 2009;5(7):380-91.

6. Dzhala VI, Talos DM, Sdrulla DA, Brumback AC, Mathews GC, Benke TA, et al. NKCC1 transporter facilitates seizures in the developing brain. Nature Med. 2005 Nov;11(11):1205.

7. Mohamad A. Mikati and Abeer J. Hani: Neonatal Seizures In: Robert M. Kliegman, NELSON Textbook of Pediatrics; 20th edition, 2016; Elsevier;Canada:2849-2854.

8. Dulac O, Milh M, Holmes GL. Brain maturation and epilepsy. InHandbook of clinical neurology 2013 Jan 1;111:441-6. Elsevier.

9. Berg A, Jallon P, Preux P. The epidemiology of seizure disorders in infancy and childhood: definitions and classifications. In: O Dulac et al, eds, Handbook of Clinical Neurology. Pediatric Neurology, Part 1 3rd edition. Elsevier; Amsterdam, Netherlands; 2013:381398.

10. Pressler RM1, Mangum B; Newly emerging therapies for neonatal seizures. Semin Fetal Neonatal Med. 2013 Aug;18(4):216-23.

11. Kumar A, Gupta A, Talukdar B. Clinico-etiological and EEG profile of neonatal seizures. Indian J Pediatr. 2007 Jan 1;74(1):33-7.

12. Lanska MJ, Lanska DJ, Baumann RJ, Kryscio RJ. A population-based study of neonatal seizures in Fayette County, Kentucky. Neurol. 1995 Apr 1;45(4):724-32.
13. Omene JA, Longe AC, Okolo AA. Seizures in the Nigerian neonate: Perinatal factors. Int $\mathrm{J}$ Gynecol Obstet. 1981 Aug;19(4):295-9.

14. Park W, Kim DY, Jung CZ, Kim SD. Clinical study of neonatal seizure. J Korean Child Neurol Soc. 1998 Oct $1 ; 6(1): 71-82$.

15. Minchom P, Niswander K, Chalmers I, Dauncey M, Newcombe R, Elbourne D, et al. Antecedents and outcome of very early neonatal seizures in infants born at or after term. BJOG An Int J Obstet Gynaecol. 1987 May;94(5):431-9.

16. Saliba RM. Risk Factors for Neonatal Seizures: A Population-based Study, Harris County, Texas, 19921994. Am J Epidemiol. 2001 Jul 1;154(1):14-20.

17. Calciolari G, Perlman JM, Volpe JJ. Seizures in the Neonatal Intensive Care Unit of the 1980s: Types, Etiologies, Timing. Clin Pediatr (Phila). 1988 Mar 1;27(3):119-23.

18. Rodney A. Radtke. Pharmacokinetics of Levetiracetam. Epilepsia. 2001;42(Suppl 4):24-27.

19. Khan O, Chang E, Cipriani C, Wright C, Crisp E, Kirmani B. Use of intravenous levetiracetam for management of acute seizures in neonates. Pediatr Neurol. 2011Apr;44(4):265-9.

20. WHO. How to investigate drug use in health facilities: selected drug indicators, action program on essential drugs (DAP), Geneva, 1993:12-25. Available at: http://apps.who.int/medicinedocs/en/d/Js2289e/.

21. Warrier I, Wei D, Natarajan G, Vali S, Aranda J. Patterns of drug utilization in a neonatal intensive care unit. J Clin Pharmacol. 2006 Apr;46(4):449-55.

22. Karande S, Sankhe P, Kulkarni M. Patterns of prescription and drug dispensing. Indian J Pediatr. 2005 Feb 1;72(2):117-21.

Cite this article as: Patil KA, Jeevangi SR, Sharanabasappa. Prospective observational study of drug utilization in neonatal seizure at a tertiary care hospital with Pharmacoeconomics. Int J Basic Clin Pharmacol 2019;8:409-14. 